\title{
Ovarian and uterine ultrasonography in pediatric patients. Pictorial essay.
}

\section{Carmen Asăvoaie ${ }^{1}$, Otilia Fufezan², Mihaela Coșarcă ${ }^{3}$}

${ }^{1}$ Emergency Children's Hospital, $1^{\text {st }}$ Pediatric Clinic, Radiology Deparatment, ${ }^{2} 3^{\text {rd }}$ Pediatric Clinic, Radiology Deparatment, ${ }^{3}$ Pediatric Surgery and Orthopedics Clinic, Cluj-Napoca, Romania

\begin{abstract}
Ultrasonography represents the method of choice in the investigation of the female pediatric pelvis. While the investigation itself poses no real challenges, an accurate interpretation of the images must take into consideration the specific features of the ovaries and uterus at certain ages. The present essay aims to demonstrate the normal appearance of the female pelvis and the changes that occur during the various stages of development as well as the some of the most common pathological conditions.
\end{abstract}

Keywords: ovary, uterus, ultrasonography, puberty, pediatric

\section{Introduction}

Pelvic ultrasonography (US) is the most useful and commonly used imaging method in the assessment of the ovaries and uterus during childhood. The investigation must always be performed considering the developmental changes which occur around puberty and the aspect of these organs at certain ages.

This pictorial essay will illustrate 1) the ultrasonographic appearance of the female internal genital organs during each developmental stage and 2) the ultrasonographic findings of some of the most frequently encountered ovarian and uterine conditions.

\section{Investigation technique}

For an optimal US visualization of the uterus and ovaries the patient must present with a well-filled, but not

Received 10.02.2014 Accepted 30.03.2014

Med Ultrason

2014, Vol. 16, No 2, 160-167

Corresponding author: Carmen Asăvoaie

$1^{\text {st }}$ Pediatric Clinic, Radiology Department

68 Moţilor street

Cluj-Napoca, Romania

E-mail: carmen.asavoaie@gmail.com over-distended bladder. Transabdominal convex or linear transducers are usually used for the examination. Transvaginal probes may be used when necessary, but only in sexually active teenagers. Also certain pathologies may benefit from a transperineal approach (urogenital malformations, hydrometrocolpos, anal atresia, etc) $[1,2]$.

In newborns and infants it is advised to use higher frequency probes (5-9 MHz), while in older children and adolescents 3-5 MHz probes are more adequate. The US exploration must provide an optimal evaluation of the anatomy, the shape, size, echogenicity, structure and vascularity of the uterus and ovaries. In some cases the use of even higher frequencies (10-12 MHz) permits a more detailed description of the echostructure, distinguishes the presence of cystic components, of wall thickening or calcifications. The Doppler interrogation is always recommended since it brings additional information about certain lesions, thus complementing the diagnosis [2].

\section{Normal aspects of the female pediatric pelvis}

\section{Uterus}

During the neonatal period the uterus appears large, has a spade-shaped appearance with a prominent cervix (fundus to cervix ratio 1:2) due to the influence of the 
maternal and placental hormones. The maximum length of the uterus is $34 \mathrm{~mm}$, the thickness can be as much as $14 \mathrm{~mm}$ and there is often an echoic endometrial lining present [1] (fig 1).

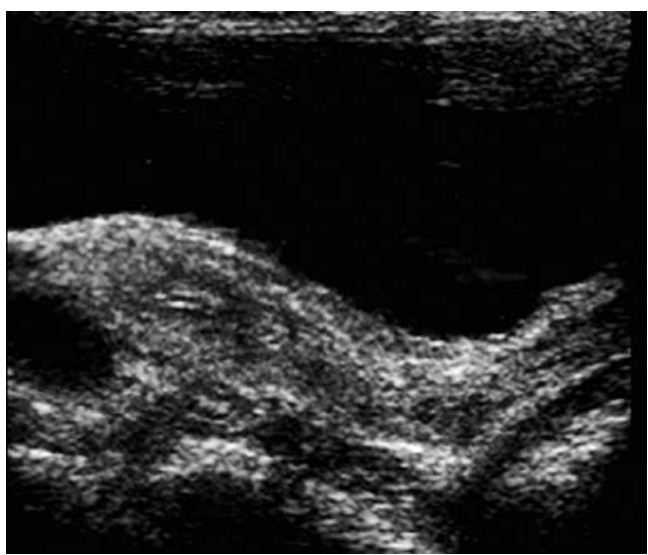

Fig 1. Normal US appearance of the uterus in a 10 months old infant. Length $30 \mathrm{~mm}$, volume $0.6 \mathrm{ml}$, fundus to cervix ratio $1: 1$, echoic endometrial line inside.

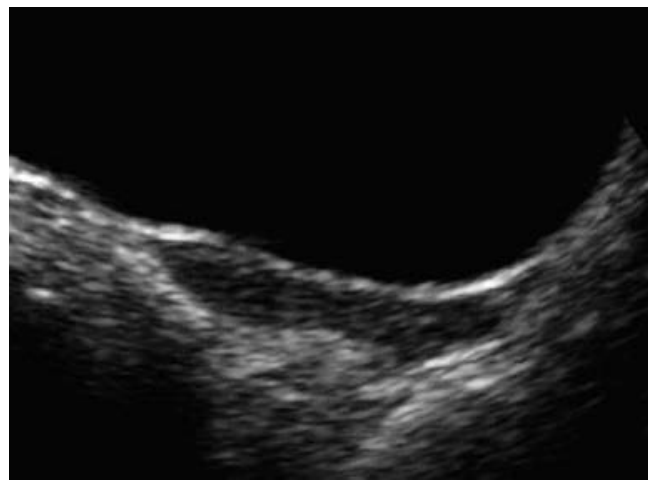

Fig 2. Prepubertal uterus: tubular shape, long axis $50 \mathrm{~mm}$, volume $2.5 \mathrm{~cm}^{3}$.

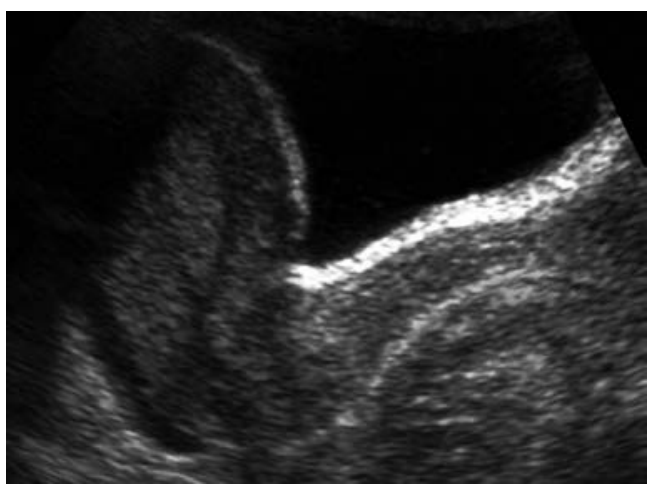

Fig 3. Pubertal uterus: pear-shaped, length $80 \mathrm{~mm}$, volume $52 \mathrm{~cm}^{3}$, fundus to cervix ratio $2: 1$, endometrium is visible, thick and echoic.
In toddlers and preschoolers both the uterus and ovaries decrease in size and volume and are rather constant until about the age of 6 when they start to grow, but significant changes in size and shape will occur after 8 years old. Before puberty begins to set in, the normal uterus has a tubular shape, with a 1:1 fundus to cervix ratio. The length of uterus is about $25-40 \mathrm{~mm}$, is $10 \mathrm{~mm}$ or less thick and the endometrial line is usually not seen at this age (fig 2).

The pubertal uterus will slowly start to resemble the adult uterus, having a pear shaped appearance (fundus to cervix ratio $2: 1$ or $3: 1$ ). After menarche the endometrium is also seen and it's thickness varies with the phases of the menstrual cycle. The uterine measurements after puberty are: 50-80 $\mathrm{mm}$ long, $30 \mathrm{~mm}$ wide and $15 \mathrm{~mm}$ thick [1] (fig 3 ).

\section{Ovaries}

In neonates ovaries are also larger, with multiple follicles inside and a volume slightly over $1 \mathrm{~cm}^{3}$, but it can

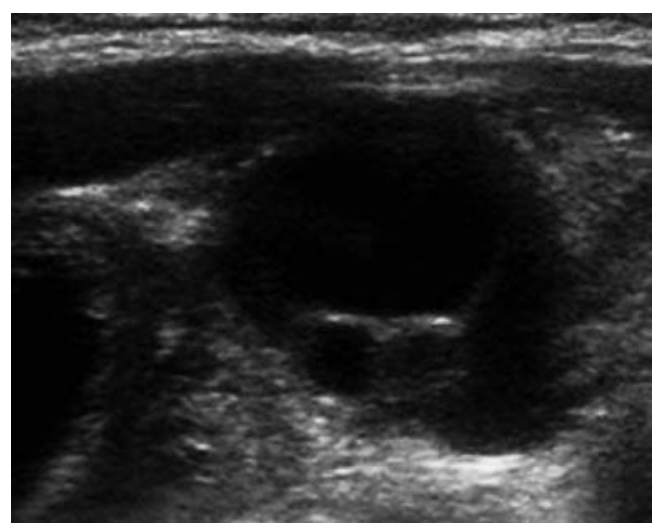

Fig 4. Large right ovary with multiple follicles in a 4 weeks old baby-girl. Ovarian volume $1.8 \mathrm{~cm}^{3}$, largest follicle $10 \mathrm{~mm}$. Image was acquired with a linear transducer $(8 \mathrm{MHz})$

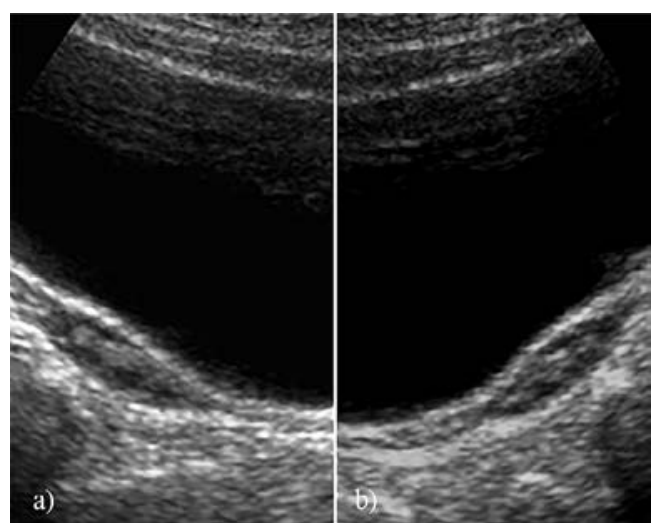

Fig 5. Normal ultrasonographic aspect of the ovaries in a three years old girl a) right, b) left: both ovaries are less than $1 \mathrm{~cm}^{3}$ and contain just a few small follicles. 
be anywhere between 1 and $3.6 \mathrm{~cm}^{3}$ [1,2] (fig 4). The ovarian volume before 6 years old is under $1 \mathrm{~cm}^{3}$ and the ovaries may show small follicles. Actually the presence of small, microcystic follicles ( $<9 \mathrm{~mm}$ diameter) is considered normal throughout childhood and they are found on sonograms in the vast majority of cases (fig 5 ). After puberty the mean volume of the mature ovary is around $8 \mathrm{~cm}^{3}$, but ranges anywhere between 2.5 and $20 \mathrm{~cm}^{3}$, depending on the phase of the menstrual cycle [1] (fig 6).

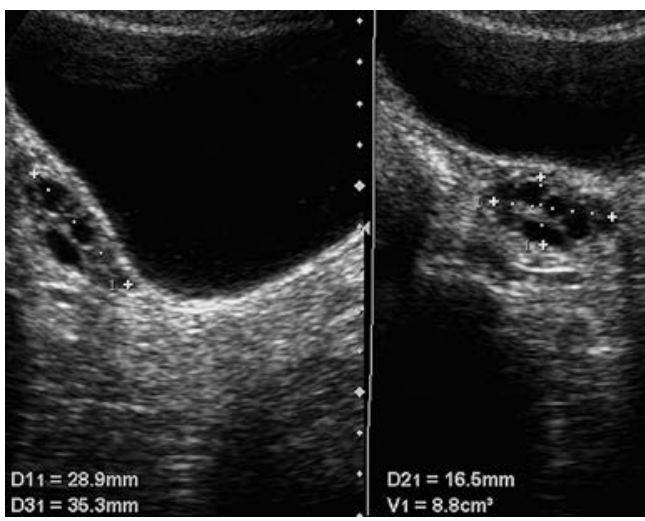

Fig 6. Normal ovary in a 14 years old teenager: ovarian volume $8.5 \mathrm{~cm}^{3}$, multiple small follicles inside.

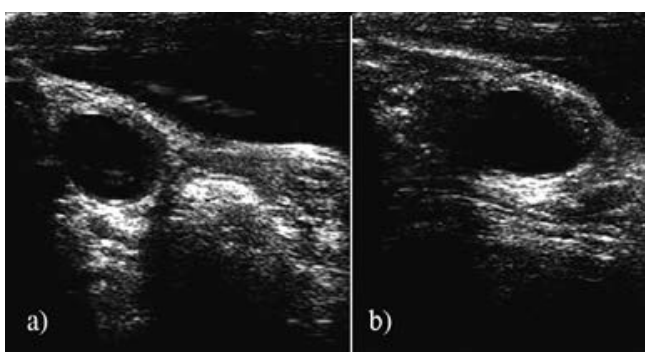

Fig 7.10 months old girl with premature thelarache. Pelvic US revealed bilateral ovarian cysts. a) right ovary, b) left ovary.

\section{Pathological aspects}

The most common indications and reasons for presentation for pelvic ultrasonography are: pelvic pain, pelvic mass, premature thelarche, adrenarche or menarche, premature or delayed puberty, primary or secondary amenorrheea, obesity, hirsutism, or sexual ambiguity.

\section{Ovarian masses}

Ovarian cysts are probably by far the most frequent finding on pelvic sonograms in girls. As previously stated, the presence of small follicles is considered normal at any age. Normal ovaries have an ellipsoid shape, are hypoechoic, homogeneous and have several, small, oval/ round, anechoic follicles.

Follicles larger than $9 \mathrm{~mm}$ before puberty are considered macrocysts and may represent one of the causes of peripheral precocious puberty (fig 7). Larger cysts after puberty onset have similar significance as in adult women. Most ovarian cysts are functional (simple, luteal, hemorrhagic cysts), develop in relationship with the menstrual cycles and require no treatment. Treatment is only indicated if the cyst does not resolve over the course of several cycles, if it is symptomatic or if it becomes complicated (torsion). A simple cyst is round and anechoic, has a thin wall, is avascular and produces acoustic enhancement (fig 8), Another type of functional cyst is the luteal cyst which develops when a corpus luteum fails to regress after ovulation. These cysts have thicker, echoic walls, which are vascularized, producing a specific appearance on the Power Doppler investigation ("ring of fire") (fig 9). If simple or luteal cysts becomes complicated by hemorrhage this usually produces pain. The sonographic appearance is represented by a complex mass, with septa, fluiddebris levels, blood clots and no signal on Doppler interrogation. All of these cysts usually resolve by spontaneously (fig 10).
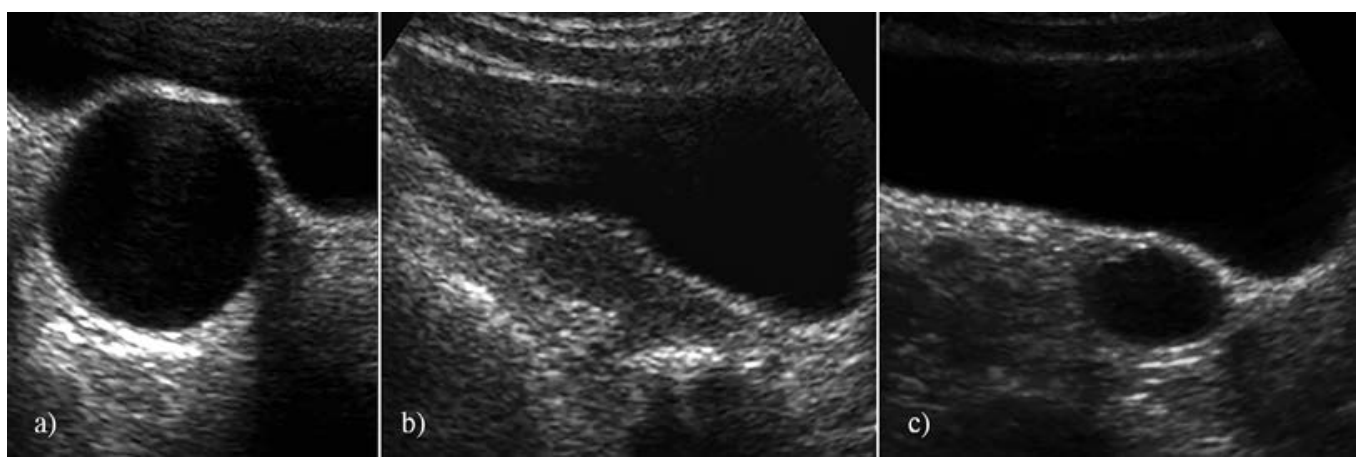

Fig 8. a) Large cystic mass found in the right ovarian fossa of a 9 years old girl with thelarche. The lesion is round, anechoic, has an unperceptible wall and produces acoustic enhancement. The uterus (b) and the left ovary (c) appear normal for age. 

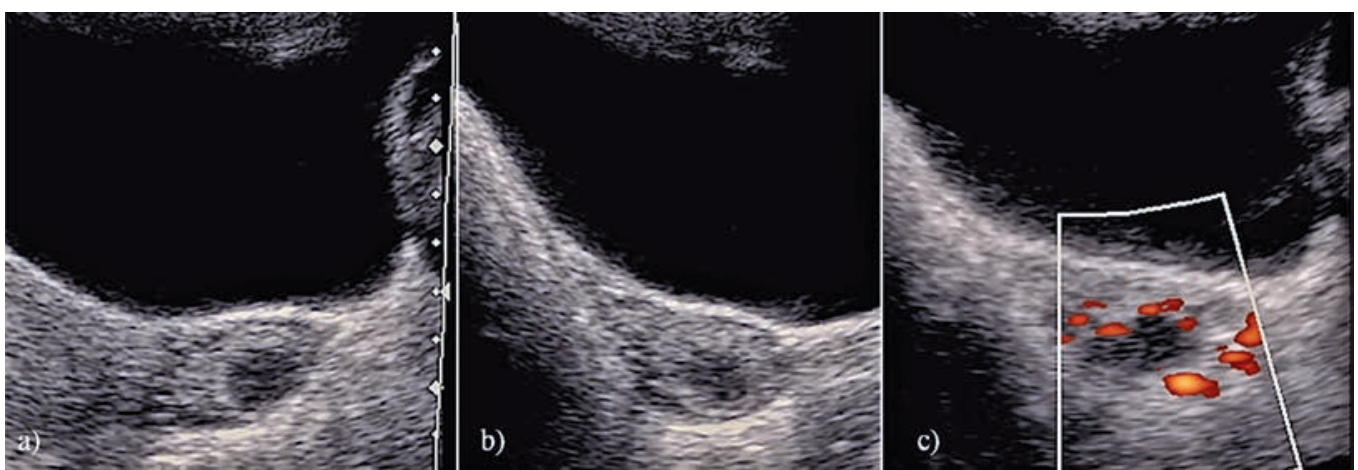

Fig 9. 15 years old teenager with left flank pain. The pelvic sonogram a) longitudinal view, b) transverse view revealed a $20 \mathrm{~mm}$ cystic mass, with impure fluid content and b) a round, vascular signal upon Power Doppler examination in the wall of the cyst (,ring of fire"). This aspect is characteristic for partially involuted corpus luteum or a luteal cyst.

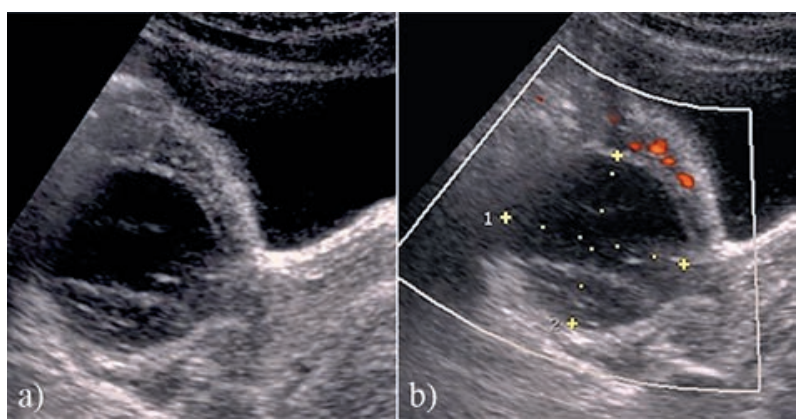

Fig 10. a) Sonographic features of a hemorrhagic cyst: large, round, inhomogeneous mass, with fluid-debris level, no vascularity (b) and acoustic enhancement in a 16 years old teenager with right pelvic pain.
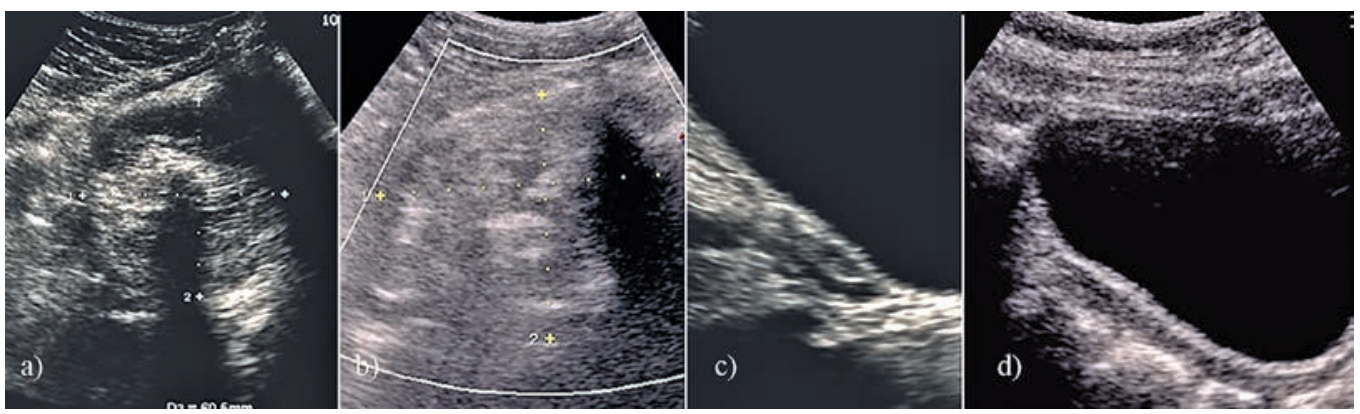

Fig 11. 10 years old girl presented with distended abdomen and a palpable pelvic mass. US revealed a large, inhomogeneous mass, occupying almost the entire pelvis, from the umbilicus down to the bladder (a). The lesion was evaluated with difficulty by US since it contained hyperechoic areas which produced intense shadowing. There was no flow detected by color Doppler inside the mass (b). It was difficult to determine the origin of the tumor, but US gave important clues towards it. The left ovary (c) was visualized and it appeared normal for age, as well as the uterus, but the right ovarian fossa was empty (d). Therefore the suspicion of a teratoma of the right ovary was raised. 
Solid or mixed masses, either benign or malignant, can also be found among the pediatric patients. Teratomas, for instance, are usually diagnosed in adolescence, but may be found at any age. They are usually quite large at presentation, can be cystic, solid or mixed masses depending on the content. The characteristic US findings are represented by a large cystic mass, with a hyperechoic solid, mural nodule, called a Rokitansky nodule or dermoid plug. In some cases, due to the size of the mass, it is difficult to asses the lesion by sonography alone and further imaging is necessary. [1,2] (fig 11, fig 12).

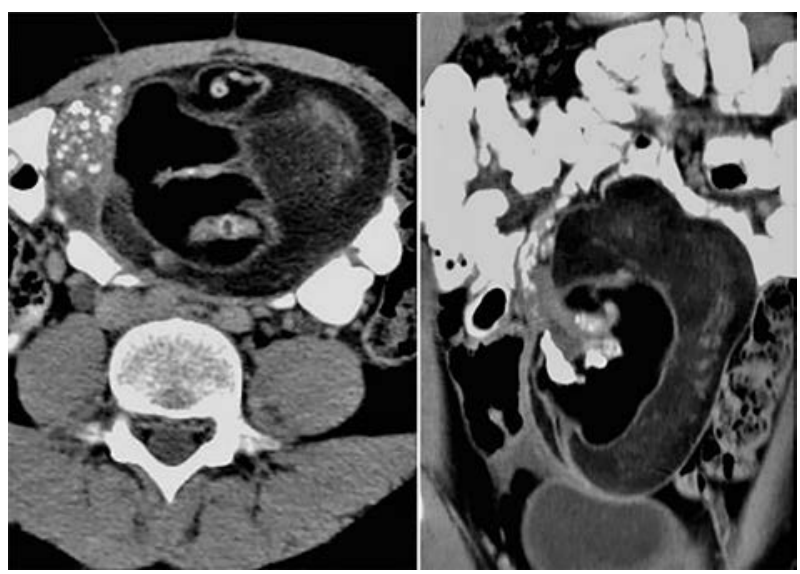

Fig 12. Contrast enhanced abdominal CT revealed a large midline pelvic mass, which extended cranially towards the iliac bifurcation, dislocated the small bowel to the sides and had contact with the right iliac vessels and the right ureter. The lesion was well-circumscribed, but highly inhomogeneous, with both solid, fluid and fat components, as well as large "pop-corn" calcifications. The left ovary appeared normal, while the right ovary was not detected. The aspect pleaded for a teratoma (dermoid cyst), which was later confirmed by the anatomopathological evaluation.

\section{Precocious puberty}

Precocious puberty is defined as the onset of sexual characteristics before the age of 8 . Central precocious puberty is the true precocious puberty, is always isosexual and is characterized by the hypothalamic-pituitarygonadal activation with consecutive sexual development and maturation. Peripheral precocious puberty or pseudopuberty is gonadotropin independent, may be both iso or heterosexual and is more common. The underlying causes of pseudopuberty are numerous and represent a very homogeneous group: ovarian cysts or other ovarian masses, adrenal masses, congeital adrenal hyperplasia (21- hydroxylase deficiency), HCG-secreting tumors, severe hypothyroidism, environmental factors [3].

On pelvic ultrasound the length of the uterus appears increased for age and if the condition has been persisting for long enough it begins to have a pear shaped appearance and the endometrium becomes visible (fig 13). The ovaries are also enlarged for age, with numerous follicles or even cysts. Doppler ultrasonography presents one of the earlier signs of precocious puberty, a broad systolic waveform with a positive diastolic flow [2]. Associated with the sonographic changes these patients also present with an increased bone age.

Isolated thelarche is defined as the development of the breast (glandular tissue) and isolated adrenarche as the appearance of sexual hair before the age of 8 . These are usually isolated, do not progress (or progress very slowly), the pelvic sonogram is normal (fig 14), as well as the bone age.

\section{Ambiguous genitalia}

In newborns with ambiguous genitalia US plays an important role in both identifying the Müllerian structures and the assessment of the adrenals. A combination of ambiguous genitalia, enlarged adrenals and presence

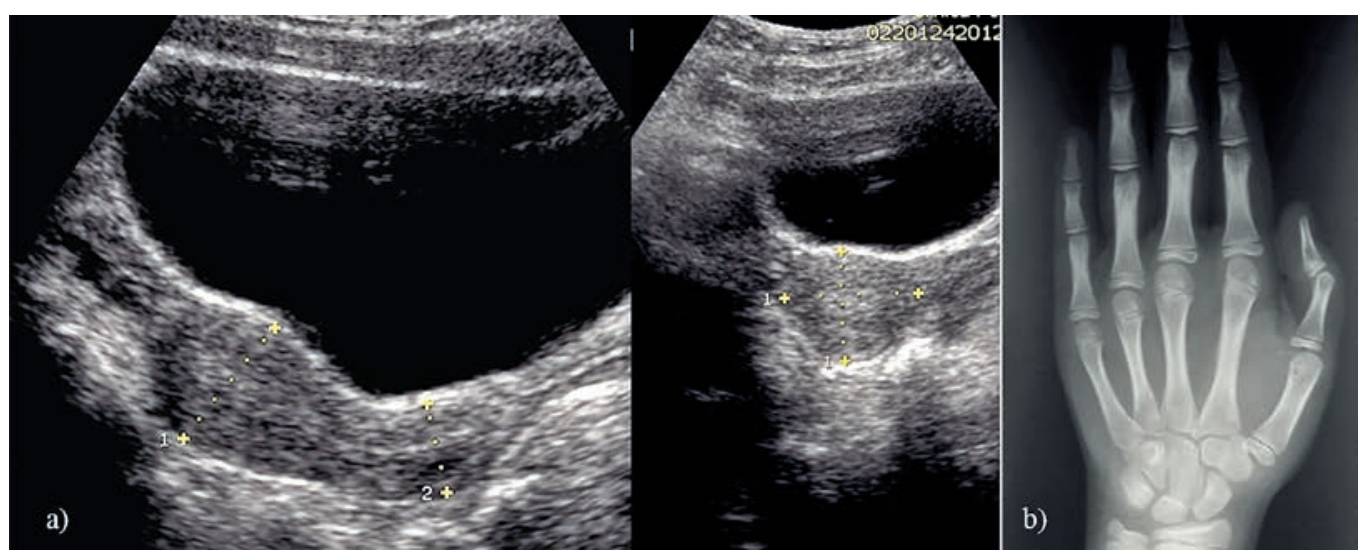

Fig 13. a) 7 years old girl with signs of premature puberty. Pelvic US reveals an enlarged, pear shaped, adult looking uterus (diameter of the uterine body exceeds that of the cervix) with an echoic endometrial band inside; b) Left hand $\mathrm{x}$ ray reveals advanced bone age (10 years old according to Greulich\&Pyle). 

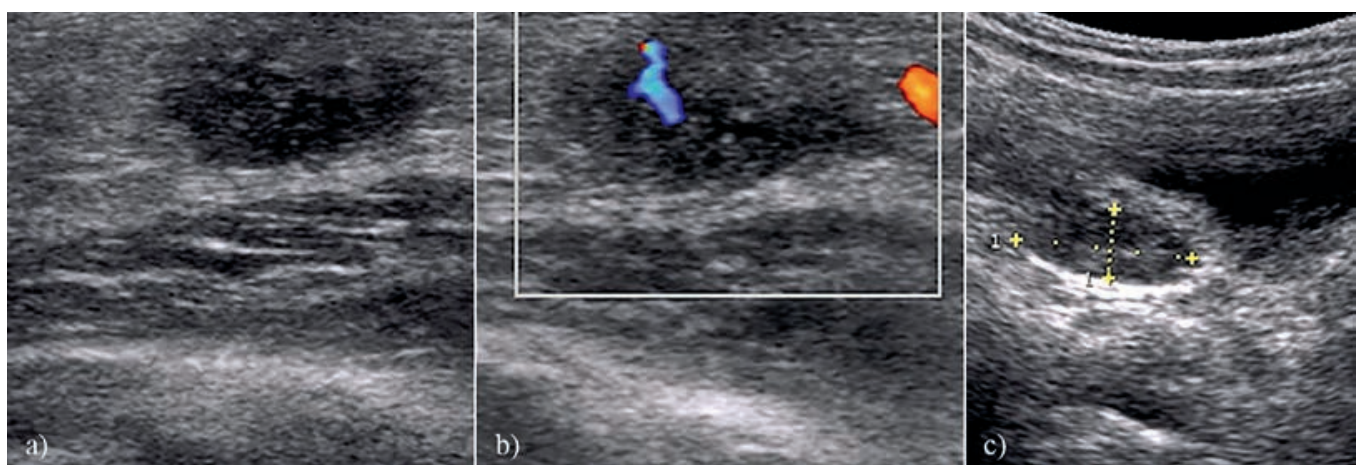

Fig 14. Three and a half years old with isolated unilateral thelarche. Breast US revealed a hypoechoic, vascularized nodule suggestive for breast tissue on the right side $(a, b)$. Pelvic sonogram revealed normal ovaries (volume $0.8 \mathrm{~cm}^{3}$ ) and uterus (c).
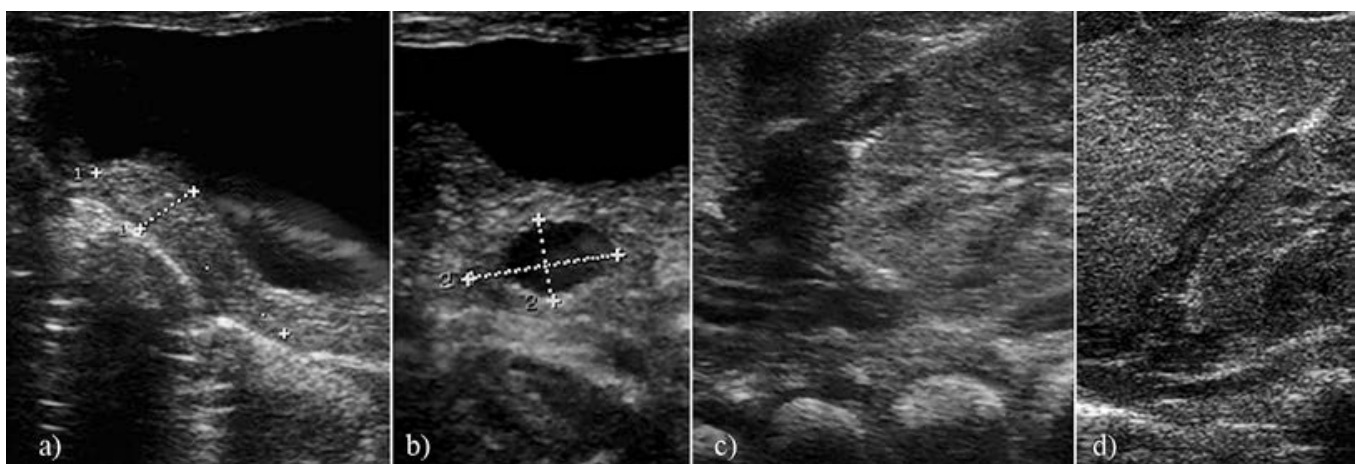

Fig 15. Eight days old with ambiguous genitalia: the pelvic US, using a linear probe, revealed the presence of female reproductive organs, normal looking ovaries and uterus (a,b); Adrenal glands present a normal US appearance, but an increased size for age $(c, d)$. The combination of ambiguous genitalia, enlarged adrenals and the presence of ovaries and uterus in the pelvis is highly suggestive for congenital adrenal hyperplasia.

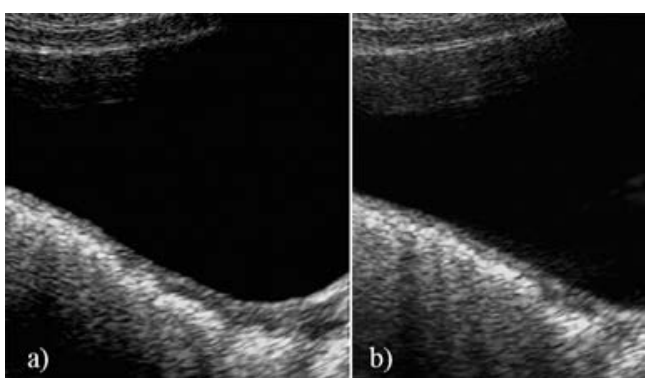

Fig 16. Eleven years old girl with short stature and no signs of puberty. a) Pelvic US revealed a hypoplastic uterus (tubular shape, $40 \mathrm{~mm}$ long and $0.4 \mathrm{~cm}^{3}$ volume and b) no ovarian structures. The suspicion of Turner syndrome was raised and genetic analysis confirmed it.

of a uterus and ovaries, detected on US, is considered to almost pathognomonic for congenital adrenal hyperplasia (CAH) [2] (fig 15).

\section{Amenorrhea}

Amenorrhea represents one of the most common indications for pelvic ultrasound in teenagers. It can

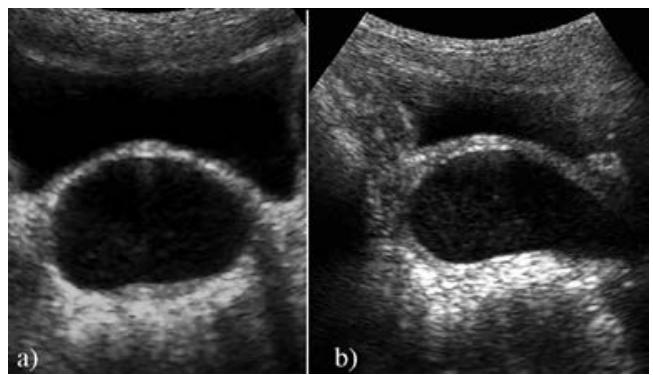

Fig 17. a) Transversal and b) longitudinal pelvic sonogram of a 6 years old girl showed a large quantity of impure fluid inside the uterine cavity. The aspect was interpreted as hydrometrocolpos due to imperforate hymen.

be primary or secondary and its causes cover a rather large spectrum (male pseudohermaphroditism, forms of congenital adrenal hyperplasia, Turner syndrome, MRKH syndrome, Prader-Willi syndrome, etc) (fig 16). In primary amenorrhea the role of US is to determine the presence of the uterus and ovaries and to 


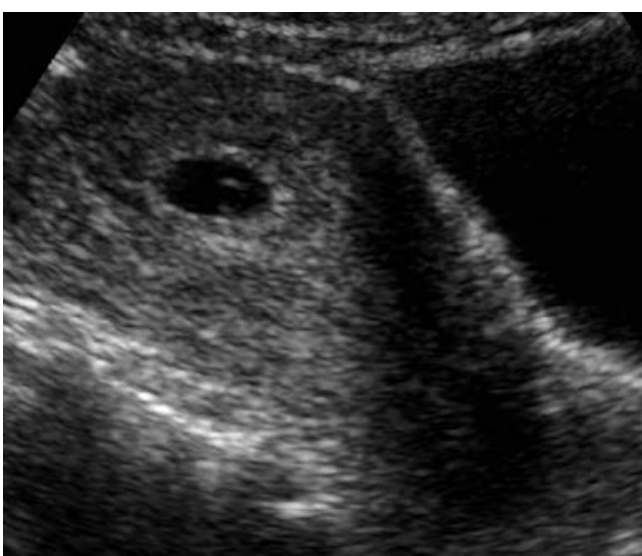

Fig 18. In 17 years old teenager with 6 weeks of amenorrhoea and nausea US revealed the presence of a evolutive pregnancy. Urine pregnancy test was also positive.

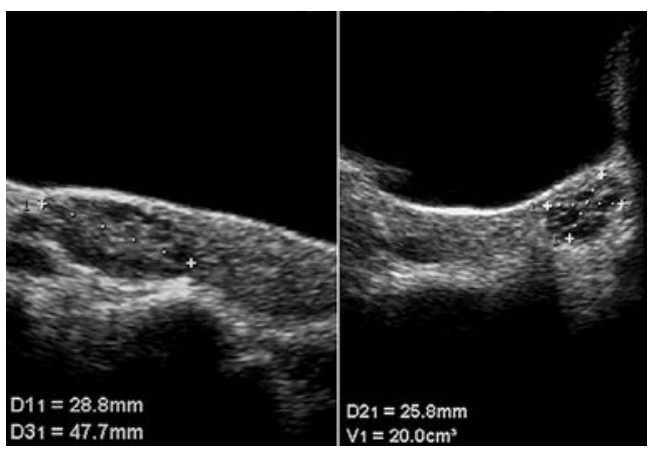

Fig 19. Longitudinal and transversal scan of the right ovary: multiple, small follicles inside, predominantly towards the periphery of the ovary in a 18 years old with amenorrhoea. The same aspect was found in left ovary. Biochemical tests were also consistent with polycystic ovary syndrome.

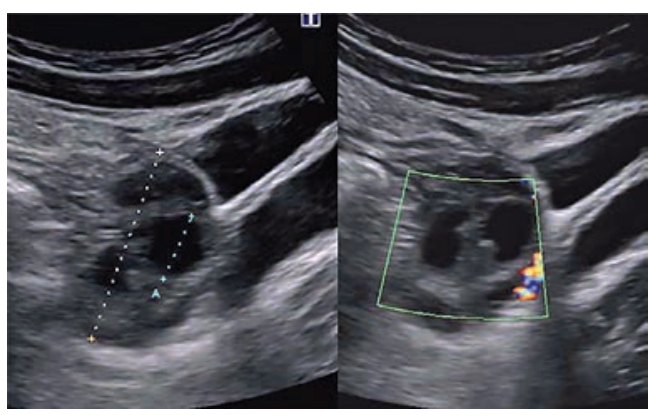

Fig 20. A 14 years old teenager with intense pain in the left pelvis: US revealed a large, echoic ovary on the left side ( 2 times larger than the right), with a few normal follicles inside, no flow on the Doppler examination, free fluid and increased echogenicity of the surrounding fat. Intraoperatory findings confirmed the suspicion of left ovarian torsion. determine if their size and appearance is according to age. In some cases US is also able to detect the cause of amenorrhea (imperforate hymen with hematometrocolpos - fig 17).

A diagnosis that must always be considered in any teenager with secondary amenorrhea is pregnancy (normal and ectopic). The US aspect obviously depends on the age of the pregnancy (fig 18). Other causes of secondary amenorrhea include: eating disorders, hyperprolactinemia, polycystic ovary syndrome (PCO), or various tumors.

PCO is a common cause of amenorrhea and is sometimes associated with obesity and hirsutism (Stein-Leventhal syndrome). The US findings include: large ovaries (1.5-3 times larger than normal) with multiple, small follicles (12 or more) and increased echogenicity (fig 19). However the diagnosis of the PCO must be confirmed through biochemical markers [2].

\section{Ovarian torsion}

Ovarian torsion is more common in the childbearing age group, but it can occur both in adolescents as well as in premenarchal girls, either due to the hyperlaxity of the ovarian pedicle or to the presence of an adnexal lesion (ovarian or paraovarian cyst). The history and clinical presentation are often nonspecific and therefore the diagnosis may be a challenge for the clinician, requiring ultrasonographic confirmation. Pelvic ultrasonography is the primary method for the diagnosis. The most constant finding US is represented by the presence of an enlarged, hypo or hyperechoic ovary, with the follicles often displaced towards the periphery of the ovary. Also there is almost always free pelvic fluid found. The changes detected by Doppler ultrasonography are also very important, but they are not always present. The most common Doppler finding is the absence of the venous flow, the arterial flow can be also absent and the diastolic flow may be absent or reversed. These signs are very useful when present, but one must always remember that a normal vascularity alone does not exclude ovarian torsion [4] (fig 20).

\section{Conclusions}

The uterus and ovaries become involved in many types of pathologies throughout infancy, childhood and adolescence. Ultrasonography has proved to be the leading imaging technique in the diagnosis and follow-up of the various conditions involving the pelvic organs. It is easily accessible, it is well tolerated by the patients, does not use ionizing radiation and more important it is able to establish the diagnosis in most situations. The examiner must be familiar with the expected appearance of these 
organs at different ages in order to issue an accurate interpretation of the findings.

\section{References}

1. Garel L, Dubois J, Grignon A, Filiatrault D, Van Vliet G. US of the pediatric female pelvis: a clinical perspective. Radiographics 2001; 21: 1393-1407.
2. Stranzinger E, Strouse PJ. Ultrasound of the pediatric female pelvis. Semin Ultrasound CT MR 2008; 29: 98-113.

3. Chung EM, Biko DM, Schroeder JW, Cube R, Conran RM. From the radiologic pathology archives: precocious puberty: radiologic-pathologic correlation, Radiographics 2012; 32: 2071-2099.

4. Poonai N, Poonai C, Lim R, Linch T. Pediatric ovarian torsion: case series and review of the literature. Can J Surg 2013; 56: 103-108. 